\title{
¿Qué implica una teoría evolutiva de las emociones respecto de la relación entre emoción y regulación emocional?
}

\author{
What does an evolutionary theory of emotions imply about the \\ relationship between emotion and emotional regulation?
}

Abel Wajnerman Paq*

Resumen: James Gross ha desarrollado una propuesta muy difundida según la cual la regulación emocional es un proceso reflexivo o de segundo orden que es diferente de las emociones. A su vez, Gross considera que esta propuesta es independiente de qué teoría sobre las emociones uno adopte. En este trabajo discuto esta presunta independencia. En particular, sostengo que el enfoque evolutivo de Tooby y Cosmides tiene dos implicaciones respecto de la relación entre emoción y regulación emocional: (1) los procesos comúnmente considerados como regulativos son, de acuerdo con esta propuesta, parte de la emoción misma y (2) la regulación emocional se lleva a cabo horizontalmente, es decir, entre emociones que se encuentran en el mismo nivel.

Palabras Clave: Emoción; Regulación emocional; Psicología evolutiva; Procesos reflexivos

\begin{abstract}
James Gross has argued that emotion regulation is a second-order or reflective process which is different from emotion. Also, he claims that his proposal is independent of the theory of emotions that one endorses. In this paper, I will challenge this presumed independence. I will argue that the evolutionary approach to emotions proposed by Tooby and Cosmides has two implications regarding the relation between emotion and emotion regulation: (1) the processes normally considered as regulatory are, under this approach, part of emotion itself and (2) emotion regulation is a horizontal process which consists in the interaction between different emotions at the same level.
\end{abstract}

Keywords: Emotion; emotion Regulation; Evolutionary psychology; Reflexive processes

\footnotetext{
* Licenciado y Doctor en Filosofía por la Universidad de Buenos Aires. Profesor Asistente para pregrado, magister y doctorado en Filosofía de la Universidad Alberto Hurtado, Chile, 2019. Ingreso a Carrera de Investigación en CONICET (categoría Investigador Asistente), tema: "Los nuevos desafíos de la explicación computacional en neurociencia cognitiva", 2019. Dirección electrónica: abelwajnerman@gmail.com
} 


\section{Introducción}

El estudio de la regulación emocional constituye un campo interdisciplinario de investigación relativamente reciente e incipiente. Ha habido un progreso significativo en la comprensión de las diferentes estrategias de regulación emocional y en su caracterización en los diferentes niveles cognitivos, neuronal, conductual, social, de desarrollo y evolutivo. Sin embargo, el concepto principal que define al área es difícil de caracterizar, no hay un acuerdo sólido respecto de una noción general de regulación emocional. Gross ha propuesto que los procesos regulativos son procesos reflexivos o de segundo orden que operan sobre las emociones y, por lo tanto, son diferentes de estas (e.g. Gross \& Thompson 2007). Esta caracterización es importante porque mostraría que el campo de la regulación emocional estudia un fenómeno diferente del de la emoción y por lo tanto puede constituir un área autónoma de investigación. En contra de esta propuesta, Kappas (2011) argumentó que las emociones siempre se auto-regulan por medio de diferentes mecanismos de retroalimentación. Esto implica que la emoción y la regulación emocional no son (siempre) procesos diferentes y que por lo tanto la propuesta de Gross no puede proporcionar una caracterización general de la regulación emocional.

En este trabajo, intentaré profundizar sobre la relación entre emoción y regulación emocional. En particular, exploraré las implicaciones que para este problema tiene un enfoque particular sobre las emociones. Esto es relevante por lo siguiente. Como veremos más adelante, Gross propone una caracterización prototípica de las emociones, la cual pretende dar un lugar a todos los componentes del proceso emocional. Dado que muchas concepciones de las emociones se diferencian entre sí justamente por el énfasis que ponen en alguno de estos componentes (las evaluaciones, las repuestas somáticas, el sentimiento, etc.), la concepción prototípica permitiría mostrar que la noción de regulación emocional de Gross es compatible con cualquiera de estos enfoques. Ahora bien, considero que ciertos enfoques evolutivos poseen elementos adicionales que, si los incorporamos a nuestra caracterización de las emociones, introducen modificaciones drásticas en el modo de entender la relación entre emoción y regulación emocional. 
En este trabajo me centraré en la concepción de las emociones propuesta en diversos trabajos por Tooby y Cosmides (e.g., Tooby y Cosmides 1990, Cosmides y Tooby 2000, Tooby y Cosmides 2008, Cosmides y Tooby 2013). Muy brevemente, estos autores sostienen que los elementos que comúnmente se consideran como los componentes de las emociones (cambios atencionales, cognitivos, fisiológicos, conductuales, etc.) constituyen programas que fueron seleccionados para resolver ciertos problemas adaptativos que surgieron en la historia de la evolución humana. Ahora bien, muchos de estos problemas sólo pueden resolverse si estos programas están coordinados adecuadamente (del modo en que ocurren cuando tiene lugar una emoción). Los autores no identifican a las emociones con ningún subconjunto de estos componentes o programas sino con programas de segundo orden o superordinados que llevan a cabo esta tarea de coordinación.

Argumentaré que la identificación de las emociones con programas superordinados tiene dos consecuencias relevantes. En primer lugar, desde esta perspectiva las emociones serían idénticas con los procesos que Gross considera casos de regulación emocional. Es decir, los mecanismos estudiados dentro del campo de la regulación emocional no serían en realidad casos de regulación emocional sino de emociones. En segundo lugar, sostendré que la perspectiva evolutiva motiva una caracterización de la regulación emocional que no es vertical (es decir, la influencia de un proceso de segundo orden sobre uno de primer orden) sino horizontal, en la medida en que los programas emocionales incluyen comandos para modularse mutuamente cuando deben coordinarse entre sí.

En la sección 2 introduciré el campo de regulación emocional y presentaré brevemente la discusión en torno a la distinción entre emoción y regulación emocional. En la sección 3 describiré el enfoque evolutivo de Tooby y Cosmides. Finalmente, en la sección 4 propondré los dos argumentos mencionados. La conclusión de estas consideraciones es que, dado que el enfoque que adoptemos sobre la emoción puede tener implicaciones importantes sobre cómo entendemos a la regulación emocional, la caracterización de la última requiere una previa discusión de la primera. Es decir, no se trata de decisiones teóricas independientes. 


\section{Regulación emocional y emociones de segundo orden}

En esta sección introduciré el campo de la regulación emocional. Mostraré que el marco propuesto nos lleva a trazar una distinción drástica entre la emoción y su regulación. Como veremos, la caracterización de las emociones presupuesta por este enfoque es compatible con muchas conocidas teorías de las emociones, dado que incluye todos los componentes que han sido frecuentemente enfatizados por diferentes teóricos en el área (atención, evaluación, respuesta fisiológica, experiencia, etc.). Sin embargo, indicaré (en la sección 3) que el enfoque evolutivo introduce elementos adicionales que serán particularmente pertinentes para la relación entre emoción y regulación. En particular, esta propuesta pone el foco en procesos que modulan los componentes tradicionales mencionados.

¿Por qué nos interesa la regulación emocional? Para entender el modo en que una respuesta emocional se desarrolla frente a la presencia de determinado estímulo no es suficiente con entender la naturaleza de las emociones mismas. Frecuentemente, las emociones son moduladas por diferentes tipos de procesos regulativos que pueden optimizar o empeorar la eficiencia con la que el sujeto interactúa con su entorno. El estudio de los procesos que regulan la emoción es un campo de formación relativamente reciente en el que intervienen diversas disciplinas psicológicas. A pesar de que no hay aún un consenso sólido sobre qué es la regulación de las emociones, sî hay cierto consenso respecto de los diferentes tipos de regulación emocional. Gross (2015) lleva adelante un esfuerzo por establecer un fundamento conceptual (o al menos ciertos lineamientos conceptuales) sobre la regulación de emociones, para lo cual elabora una serie de distinciones entre tipos de regulación emocional.

Gross considera que los diferentes tipos de regulación emocional se correlacionan con los diferentes componentes de la emoción. Por este motivo, es importante decir algo sobre esta última. Las emociones son episodios complejos que frecuentemente involucran una serie de elementos concatenados: Una situación externa o interna (es decir, una situación percibida o meramente imaginada o recordada) se le presenta a un sujeto. Este presta atención a ciertos aspectos de la situación, es decir, lleva a cabo un recorte atencional de la misma, enfatizando ciertos rasgos. El sujeto luego evalúa la 
relevancia de la situación respecto de sus objetivos a partir de los rasgos atendidos. Por último, esta evaluación genera una serie de cambios en la fisiología, la experiencia y la conducta del sujeto (Gross 2015). Una pregunta central en torno a la que se han articulado diferentes teorías es qué componente o conjunto de componentes de estos episodios es constitutiva de la emoción. Se trata de la pregunta sobre qué conjunto de componentes constituye las condiciones necesarias y suficientes para que ocurra una emoción.

En lugar de comprometerse con una caracterización de las emociones que enfatice un elemento o subconjunto de elementos, Gross toma a todos los componentes como constituyendo un prototipo de emoción, esto es, un conjunto de características que son típicas o diagnósticas para pertenecer a la categoría "emoción" (e.g., Gross y Thompson 2007). Un episodio prototípico de emoción es una secuencia de eventos que incluye una situación, un sesgo atencional respecto de ciertos aspectos de la misma, una evaluación a partir de dichos aspectos y una respuesta (en términos conductuales, fisiológicos y de experiencia) producida por dicha evaluación. Que un episodio cuente con alguno o varios de estos aspectos hace más probable que constituya una emoción. Esto es lo que Gross (2015) llama el "modelo modal” de la emoción. Adoptar este modelo modal mostraría que su caracterización de la regulación emocional es relativamente neutral respecto de qué teoría de las emociones uno adopte.

A partir de este modelo, Gross formula el "modelo procesual" de la regulación emocional. Este modelo distingue los diferentes tipos de regulación emocional según cuál de los diferentes componentes de la emoción (especificados por el modelo modal) tengan por objetivo. El primer tipo, la regulación de la situación, se divide en dos sub-tipos: la selección de situación y la modificación de situación. La selección de situación consiste llevar a cabo acciones que hagan más o menos probable que enfrentemos situaciones que esperamos que den lugar a emociones deseables o no deseables. Un ejemplo podría ser faltar a una fiesta a la que sabemos que irá una expareja para evitar una situación dolorosa. La modificación de situación consiste en alterar determinada situación para alterar su impacto emocional. Un ejemplo podría ser esconder obras de arte escandalosas cuando nos visitan nuestros suegros conservadores. 
Ahora bien, es posible regular las emociones sin cambiar efectivamente el entorno. Las situaciones tienen muchos aspectos y la modificación de atención es el modo en que los individuos dirigen su atención en una situación dada para influenciar sus emociones. Las dos estrategias atencionales más importantes son la distracción y la concentración. La distracción es quitar la atención de la situación, como cuando un infante que aún no tiene la capacidad motriz para alejarse de un estímulo negativo, quita la mirada del mismo (Stifter \& Moyer, 1991; Rothbart \& Sheese, 2007). La distracción también puede involucrar cambiar el foco interno, como cuando los individuos invocan pensamientos o memorias que son inconsistentes con un estado emocional no deseable (Watts, 2007). La concentración lleva a fijar la atención en las características de una situación que disparan la emoción.

Incluso después de que la situación ha sido elegida, modificada y atendida, una respuesta emocional no se sigue inevitablemente. Los cambios cognitivos son modos en que alteramos cómo evaluamos una situación en la que estamos para modificar su significado emocional. Por ejemplo, esto puede suceder cuando reevaluamos nuestra situación comparándola con la de alguien menos o más afortunado. Por último, la modulación de respuesta emocional consiste en influenciar la respuesta fisiológica, experiencial o conductual que constituye un proceso de emoción. Los modos de influenciar los aspectos fisiológicos y experienciales son comunes. Las drogas pueden ser usadas para modificar ciertas respuestas fisiológicas como la tensión muscular (los ansiolíticos) o la hiperactividad simpática (los beta-bloqueadores). El ejercicio y la relajación pueden ser usadas para disminuir los aspectos fisiológicos y experienciales de las emociones negativas, y el alcohol, los cigarrillos, las drogas e incluso la comida pueden ser usados para modificar la experiencia emocional.

Ahora bien, el modelo procesual de la regulación emocional nos da una taxonomía de estrategias de regulación, pero no nos explica aún cómo estas se llevan a cabo, cuáles son los mecanismos subyacentes a la regulación. Lo que Gross (2015, p. 9) llama "el modelo procesual extendido de la regulación emocional" nos ofrece esta explicación. Dado que el modelo es muy complejo para ser presentado detalladamente aquí, ofrezco sólo algunos lineamientos generales sobre los mecanismos de regulación emocional. Vimos que una parte importante de las emociones es el proceso de evaluación. Un conjunto de características del mundo atendidas es evaluado y esta evaluación produce una 
serie de respuestas somáticas, experienciales y conductuales. La idea central de Gross (2015) es que los mecanismos de regulación emocional son también mecanismos de evaluación pero que toman a los mecanismos de evaluación de primer orden, las emociones, como su objeto. La regulación emocional es una evaluación de segundo orden. Un mecanismo de regulación toma como input algún aspecto del mecanismo de primer orden (por ejemplo, un sesgo atencional, una evaluación o las respuestas que genera), atiende a ciertos aspectos de este input y genera una evaluación de los mismos. Esta evaluación genera una serie de respuestas (fisiológicas, experienciales y conductuales) orientadas a modificar o mantener lo representado por el input (el aspecto relevante de la emoción evaluada).

Este último aspecto de la propuesta de Gross traza una línea excluyente entre emoción y regulación emocional. Si bien presentan semejanzas relevantes (ambos son procesos que involucran evaluaciones) que presumiblemente permitan afirmar que son procesos del mismo tipo, la regulación es un proceso numéricamente diferente de la emoción. La posibilidad de establecer una distinción de este tipo entre la emoción y regulación ha sido uno de los puntos de discusión dentro del área. Por ejemplo, se ha sostenido que hay muchos casos de regulación emocional que no requieren la intervención de procesos reflexivos o de segundo orden. Esto es, las emociones frecuentemente se auto-regulan mediante diferentes mecanismos de retroalimentación. Este punto es importante porque implica que (al menos parte de) el estudio de la regulación emocional es parte del estudio de las emociones mismas. Kappas (2011) ofrece una serie de argumentos para apoyar este punto. Mencionaré sólo dos casos. En primer lugar, las emociones negativas son procesos que se anulan a sí mismos. Esto se sigue simplemente de que cuando un estímulo dado (por ejemplo, una araña) produce una emoción negativa (por ejemplo, miedo), entonces la emoción produce una conducta (por ejemplo, matar la araña, correr, etc.) que está orientada a eliminar la presencia del estímulo y, consecuentemente, de la emoción misma. Kappas afirma que todas las emociones negativas se auto-eliminan en este sentido y que esto involucra una forma de auto-regulación.

En el caso de las emociones positivas, Kappas describe un mecanismo diferente. La razón por la que no estamos persiguiendo constantemente el loop de retroalimentación positiva para permanecer en estados de emoción positiva 
es el mecanismo de saciedad. Los estados positivos tienen dos fases: una actividad apetitiva y una respuesta consumatoria (e.g., Arnold 1960). La última permite que la primera culmine. Hay mecanismos responsables del período refractario que hace terminar a la conducta apetitiva después de la consumación, por ejemplo, habiendo comido o en el contexto de la saciedad sexual post-orgásmica (ver Georgiadis \& Kortekaas, 2010). Los mecanismos neuronales de estos dos procesos son frecuentemente llamados wanting/reward y pleasure/liking (e.g., Berridge, 2009). La saciedad sería una parte del mecanismo subyacente a las emociones positivas por medio de la cual estas se regulan a sí mismas.

En la sección 4 propondré otra línea diferente para cuestionar la caracterización de Gross de la regulación emocional. Vimos que el modelo modal de la emoción permitiría mostrar que la noción de regulación emocional es neutral respecto de qué teoría de las emociones adoptemos. Sostendré que, por el contrario, cierta versión del enfoque evolutivo de las emociones tiene implicaciones relevantes en contra de la propuesta de Gross. En la siguiente sección presentaré esta propuesta.

\section{Un enfoque evolucionista}

Adoptar un enfoque evolucionista sobre las emociones constituye una vía alternativa a la de Kappas (2011) para cuestionar la propuesta de Gross. Esta estrategia no consiste en mostrar que podemos encontrar procesos regulativos en las emociones mismas, tal como las entiende Gross, sino más bien en proponer que las emociones son procesos más complejos que los descriptos por su modelo modal. Como veremos, desde una perspectiva evolucionista las emociones no son meramente una secuencia de sub-procesos aislados sino principalmente los mecanismos que los coordinan. En este sentido, las emociones involucran cierto tipo de regulación de dichos subprocesos. En lo que sigue introduciré la perspectiva evolucionista.

La psicología evolucionista es a veces considerada como una segunda fase de la revolución cognitiva. Según Cosmides y Tooby (2013), mientras que la primera fase estaba centrada en diversos mecanismos o procesos computacionales que implementan nuestras capacidades mentales (e.g, 
percepción, atención, categorización, razonamiento, memoria, etc.), esta segunda fase estudia el modo en que estos procesos son 'diseñados' por la selección natural para regular adaptativamente la fisiología y el comportamiento procesando información sobre el entorno. Esta perspectiva proporciona un nuevo modo de pensar sobre los mecanismos cognitivos. Por ejemplo, permite entender la manera en que se relacionan las diferentes capacidades mentales como una estrategia para resolver un problema adaptativo. Como veremos, esta perspectiva parece particularmente pertinente para pensar a las emociones.

La perspectiva evolucionista sostiene que los mecanismos mentales son rasgos cuya permanencia en una población depende de la selección natural. Decimos que un rasgo es seleccionado cuando esta permanencia resulta de una ventaja adaptativa (mayor éxito reproductivo) (Richardson, 1996, p. 545). Este enfoque implica que el mecanismo computacional subyacente a cada capacidad mental está diseñado por la selección natural para resolver un problema adaptativo que surgió durante la historia evolutiva humana (o anteriormente) como el reconocimiento de rostros, la búsqueda de alimento, la elección de pareja, la regulación del ritmo cardíaco, la regulación del sueño, etc. Ahora bien, la existencia de estos mecanismos crea a su vez un nuevo problema adaptativo: los mecanismos o programas que resuelven problemas específicos podrían, si son activados simultáneamente, producir outputs que interfieren o se inhiben entre sí. Por ejemplo, dormir y huir de un predador requieren acciones, computaciones y estados fisiológicos inconsistentes.

Para evitar este problema, la mente debe estar equipada con programas superordinados que permiten ignorar y desactivar algunos programas cuando otros son activados (por ejemplo, un programa que desactiva los programas de sueño cuando las rutinas para la evasión de un predador son activadas). A su vez, muchos problemas adaptativos requieren de la activación coordinada de diferentes programas. Por ejemplo, evitar un predador requiere la modulación simultánea del ritmo cardíaco y la agudeza auditiva. Para este fin, se requiere un programa que coordine estos sub-programas produciendo la configuración específica necesaria para resolver el problema relevante. Los programas que llevan a cabo esta coordinación son justamente las emociones (Tooby \& Cosmides 2008).

Según esta propuesta, una emoción es un programa superordinado cuya 
función es dirigir las actividades e interacciones de los subprogramas que constituyen la percepción, la atención, las inferencias, el aprendizaje, la memoria, la elección de objetivos, la prioridad de motivaciones, la categorización y los marcos conceptuales, las reacciones fisiológicas, etc. En contra de muchas propuestas tradicionales, como las mencionadas en la sección anterior, la emoción no se identifica con una categoría de efectos, como la fisiología, la conducta, las evaluaciones cognitivas o los sentimientos, sino que consiste en un conjunto de instrucciones para todos ellos (Tooby y Cosmides, 1990; Cosmides y Tooby, 2000; Nesse, 1990; Keltner et al., 2006, Sznycer, D, Cosmides, L y Tooby, J., 2017).

Por ejemplo, el miedo a los predadores es un sistema neurocognitivo que coordina una serie de mecanismos para formar una configuración que permite evitar predadores eficientemente. Sincroniza una serie de componentes, ajustando los parámetros y umbrales de unos y apagando otros. El miedo incrementa la activación del sistema de atención que monitorea animales, de los mecanismos que rastrean rutas de escape, los marcos conceptuales que caracterizan localizaciones como seguras o peligrosas, los motores de búsqueda que permiten acceder a información sobre animales peligrosos y los procesos fisiológicos que facilitan el escape o el ataque, modifica los umbrales de detección auditiva y activa los sistemas de inferencias relevantes para distinguir el sonido del viento del de un león al acecho. A su vez, el miedo desactiva los mecanismos responsables del cortejo, la búsqueda de alimento, o el sueño, y cualquier otro que pueda interferir con la evasión de un predador. Toda emoción es un programa seleccionado para controlar una serie de subprogramas con el objetivo de resolver un problema adaptativo ancestral de este tipo.

En la próxima sección consideraré qué consecuencias tiene una concepción de este tipo respecto de la relación entre emoción y regulación emocional.

\section{Regulación emocional desde una perspectiva evolucionista}

En esta sección presentaré dos argumentos que muestran que adoptar el 
enfoque evolucionista de las emociones conlleva dos cambios drásticos respecto del modo de entender a la regulación emocional. En primer lugar (sección 4.1) sostendré que el enfoque implica que la línea entre emoción y regulación emocional está mal trazada. Los tipos de procesos que se considera que constituyen el dominio de la regulación emocional son en realidad casos de emociones. En segundo lugar (sección 4.2) argumentaré que la propuesta de Tooby y Cosmides mina la motivación para entender a la regulación como un proceso reflexivo en el que la emoción es controlada por un proceso de orden superior porque su caracterización de las emociones implica que la regulación de las mismas se da de manera lateral. Esto es, las emociones cuentan con mecanismos para regularse entre sí de la manera más conveniente para la supervivencia.

\subsection{Emoción como coordinación de estrategias regulativas}

Vimos que, según el enfoque evolucionista, una emoción es un programa o un mecanismo que regula (en el sentido de que activa o inhibe) ciertos programas o mecanismos de primer orden. Esto en sí mismo no implica nada respecto de la distinción entre emoción y regulación emocional. El hecho de que las emociones regulan procesos cognitivos es compatible con que ellas sean a su vez reguladas por otros procesos diferentes de ellas.

Ahora bien, podemos ver que la propuesta evolutiva sí tiene implicaciones relevantes respecto de la noción de regulación emocional si nos enfocamos en los procesos específicos que son regulados por las emociones. Los mecanismos de primer orden que son regulados por el programa emocional son justamente los que vimos que Gross considera componentes prototípicos de la emoción. Los programas superordinados regulan los estados atencionales, las evaluaciones y las respuestas fisiológicas, de experiencia y conductuales, es decir, los tipos de estados que se consideran comúnmente constitutivos de la emoción. Vimos que el miedo, por ejemplo, modula la atención centrándola en aspectos del entorno relevantes para la respuesta requerida, como animales y rutas de escape, modula la evaluación también de manera acorde a la situación, cambiando el tipo de situaciones que se caracterizan como seguras o peligrosas y, por último, regula los procesos 
fisiológicos que facilitan el escape o el ataque.

¿Qué implica este hecho respecto de la relación entre emoción y regulación emocional? Antes de responder esta pregunta, es importante notar qué cosa no implica. En particular, esto no implica que las emociones se regulen a sí mismas. Aunque los componentes regulados son típicamente considerados los constituyentes de la emoción, este no es el caso de la perspectiva evolutiva. Como vimos, la emoción se identifica dentro de esta propuesta con el programa que regula dichos componentes y no con los componentes mismos. Lo que las emociones regulan es algo diferente de ellas mismas, no es una parte constitutiva de ellas.

La consecuencia que sí tiene este enfoque está relacionada con qué procesos particulares pueden ser identificados como casos de regulación emocional y qué procesos pueden ser identificados como emociones. El programa superordinado que el enfoque evolutivo identifica con la emoción está constituido por los mecanismos o procesos que según el enfoque de Gross serían casos de regulación emocional. El miedo, por ejemplo, es el proceso de modular de determinada manera (entre otras cosas) la atención, la evaluación y las respuestas cognitivas. Es decir, el miedo está constituido (entre otras cosas) por instancias de modificación atencional, cambio cognitivo y modulación de respuesta.

Veamos este punto con un poco más de detalle. Vimos que el miedo a los predadores coordina una serie de procesos para poder evitar eficientemente a los predadores. En primer lugar, este programa modula la atención de diferentes maneras. Una de las estrategias atencionales es la concentración, que lleva a fijar la atención en las características de una situación. El miedo lleva a enfocar la atención en, por ejemplo, la conducta de otros animales y en posibles rutas de escape. La otra estrategia atencional, la distracción (esto es, es quitar la atención de ciertos aspectos de una situación) también plausiblemente activada por el miedo. Vimos que el miedo desactiva los mecanismos que pueden interferir con la evasión de un predador (por ejemplo, los mecanismos de cortejo y de búsqueda de alimento). Es plausible pensar que este proceso involucra quitar la atención de los estímulos que activan estos mecanismos.

A su vez, el miedo involucra la estrategia de cambio cognitivo, es decir, modificar el modo en que evaluamos una situación. Vimos que esta emoción 
modula los marcos conceptuales que caracterizan localizaciones como seguras o peligrosas. Por ejemplo, caminos familiares y confortables pasan a ser evaluados como peligrosos y lugares raros que normalmente no ocuparíamos (e.g., las ramas de un árbol) pasan a ser considerados lugares seguros (Tooby y Cosmides 2008). Es decir, el miedo produce un cambio cognitivo respecto de diversos aspectos del entorno. A su vez, vimos que el miedo hace más accesible información relevante para evaluar otros animales (si son peligrosos, si podrían cooperar, etc.). La disponibilidad de esa información puede facilitar evaluaciones que en otro contexto no llevaríamos a cabo.

Por último, el miedo involucra la estrategia de modulación de respuesta (esto es, influenciar la respuesta fisiológica, experiencial o conductual). Vimos que el miedo activa procesos fisiológicos que facilitan el escape o el ataque. Algunos de los procesos mencionados por Tooby y Cosmides (2015) incluyen: La mucosa gástrica se vuelve blanca en tanto la sangre abandona el tracto digestivo (un proceso concomitante al hecho de que la alimentación deja de ser una prioridad), la adrenalina se dispara, el ritmo cardíaco puede aumentar o subir (dependiendo de si la situación requiere huir o quedarse inmóvil), la sangre fluye hacia la periferia, y se envían instrucciones a los músculos (Cannon, 1929; Tomaka, Blascovich, Kibler y Ernst, 1997; Ekman, 1982).

Estas consideraciones muestran que el miedo está constituido por los procesos que Gross identifica como instancias de regulación emocional. Este hecho implica que, si el enfoque evolutivo es el enfoque correcto o adecuado respecto de las emociones, entonces la línea que divide al estudio de la emoción del estudio de la regulación emocional está mal trazada. Los procesos que comúnmente se estudian dentro del campo de la regulación emocional serían en realidad instancias de emociones.

Por supuesto, algo puede ser al mismo tiempo una instancia de emoción y de regulación emocional. Esto es lo que muestran los casos de autoregulación mencionados por Kappas (2011). Sin embargo, como vimos más arriba, según el enfoque evolutivo los procesos regulados por las emociones no son a su vez emociones. Es decir, las emociones entendidas como programas superordinados no son procesos de regulación de emociones. De esta manera, esta propuesta implica que los procesos estudiados dentro del campo de la regulación emocional son en realidad instancias de emociones y no de 
regulación emocional.

Otra consecuencia importante de este enfoque es que no sólo modifica cómo trazamos la línea entre emoción y regulación emocional sino también cómo trazamos las líneas entre diferentes procesos regulativos. Si bien las emociones son identificadas con los procesos regulativos de Gross, cada tipo de emoción no se identifica con ningún tipo de estrategia particular. Como vimos en el caso del miedo, las emociones involucran combinaciones de los diferentes tipos de estrategias. Las diferentes estrategias son vistas y estudiadas desde esta perspectiva como componentes de un mismo mecanismo y no como mecanismos aislados.

En resumen, el enfoque evolutivo involucra un cambio tanto respecto de qué procesos consideramos como casos de regulación emocional como respecto del modo de distinguir entre procesos regulativos.

\subsection{Regulación emocional como emoción de primer orden}

Vimos en la sección anterior que el enfoque evolutivo implica que los tipos de procesos que son objeto de estudio dentro del campo de la regulación emocional serían en realidad componentes de la emoción misma y no de su regulación. Ahora bien, vimos que esto implica sólo que la línea que divide el estudio de la emoción del de su regulación está mal trazada y no que tal línea no exista. Una manera intuitiva de volver a trazar dicha línea podría ser aplicar a la concepción de las emociones de Tooby y Cosmides la idea de Gross de que la regulación emocional es un proceso de control reflexivo.

En esta línea, podríamos afirmar que regulación es un programa reflexivo que regula a los programas superordinados que constituyen las emociones. Este sería un programa de tercer orden ya que tiene por objeto a las emociones que, como vimos, constituyen programas de segundo orden. La motivación de esta propuesta podría darse a partir de reproducir el argumento de Tooby y Cosmides para justificar la necesidad de programas de segundo orden. Así como los mecanismos o programas de primer orden que resuelven problemas específicos como dormir y huir podrían producir outputs que interfieren o se inhiben entre sí si se activan simultáneamente, podría surgir 
también un problema de coordinación en la activación de programas superordinados. Por ejemplo, supongamos que un animal se encuentra con un organismo que podría ser evaluado o bien como un peligro o bien como una presa. Estas diferentes evaluaciones podrían motivar la activación de diferentes programas emocionales que son incompatibles entre sí, en tanto uno requiere activar los programas de primer orden que faciliten la huida mientras que el otro requerirá activar los que faciliten capturar una presa. La regulación emocional podría ser el programa de tercer orden que lleva a cabo la decisión de activar uno de estos dos programas emocionales y desactivar el otro.

Considero que este tipo de argumento no puede apoyar la existencia de procesos regulativos de tercer orden. La razón es que los programas emocionales ya cuentan con las herramientas para tomar este tipo de decisiones por sí mismos. Veamos esto con un poco de detalle. Según Tooby y Cosmides, los programas emocionales dependen de inputs externos específicos. Por ejemplo, cuando un organismo está sólo a la noche, un circuito de detección determina que uno ha entrado en la situación de "posible acecho y emboscada" a partir de pistas que indican la posible presencia de un predador. El output de este circuito es el input del programa emocional (Tooby y Cosmides 2008). Ahora bien, supongamos que al mismo tiempo otro circuito indica la presencia de una potencial pareja sexual y que esta información constituye el input para un programa alternativo e incompatible con el miedo (el programa encargado de lograr la reproducción sexual). Este tipo de casos no van a requerir de la intervención de un programa de orden superior. Los inputs incompatibles no van a resultar en un problema de interferencia porque es parte ciertos programas emocionales la capacidad de apagar otros incompatibles con ellos.

Por ejemplo, es parte del programa del miedo la modulación de los objetivos y los pesos motivacionales. En cuanto se activa este programa,

la seguridad deviene la prioridad máxima. El resto de los objetivos y los programas computacionales que los llevan a cabo son desactivados: uno no está más hambriento, dejamos de pensar cómo atraer a una potencial pareja, practicar una nueva habilidad no resulta atractivo. El foco de planeamiento se estrecha al presente, las preocupaciones respecto de ayer y mañana se desvanecen temporalmente. El hambre, la sed y el dolor son suprimidos (Tooby y Cosmides 2008, p. 118. Las cursivas son mías). 
Esto quiere decir que, en el nivel de las emociones, el problema de conflicto o interferencia que motiva la regulación se resuelve de manera lateral. Los programas emocionales mismos incluyen la capacidad de modificar las prioridades y así quitarle efectividad al input de un programa emocional alternativo que podría poner en peligro la supervivencia. El tipo de argumento que motiva la postulación de un programa superordinado no puede emplearse para introducir un programa de tercer orden, es decir, para entender a la regulación emocional como un proceso diferente de la emoción misma. Este punto implica que las emociones se regulan a sí mismas, aunque de un modo diferente al propuesto por Kappas (2011). Aquí no se trata de mecanismos de retroalimentación por medio de los que una emoción dada se regula a sí misma sino de mecanismos por medio de los cuales diferentes emociones se regulan unas a las otras (de aquí la idea de una regulación "lateral").

Adicionalmente, si analizamos la descripción propuesta de los programas emocionales podemos notar que, además de este tipo de regulación lateral u horizontal, hay cierto nivel de auto-regulación por retroalimentación dentro de este enfoque. Como vimos, las emociones requieren de cierto input que permite establecer el tipo de situación en la que se encuentra un organismo. Este elemento cumple el mismo rol que la percepción y/o la evaluación en los enfoques tradicionales: establece qué está sucediendo en el entorno y por lo tanto qué respuesta es requerida para nuestra supervivencia. Si bien vimos que la atención y las respuestas fisiológicas no son constitutivas de la emoción, sino que son reguladas por el programa emocional, este input tiene un rol diferente: le da inicio al programa emocional. Ahora bien, el programa emocional modula este circuito de detección. Veamos esto con algo de detalle.

Uno de los elementos del programa del miedo es un cambio en el "marco conceptual" responsable de activar dicha emoción. Vimos que el miedo determina una modulación de categorías como "peligroso" o "seguro". Hemos mencionado que, por ejemplo, el miedo hace más accesible información relevante para evaluar otros animales, esto es, información que permite determinar si son peligrosos, si podrían cooperar, etc. La disponibilidad de esa información puede facilitar evaluaciones que en otro contexto no llevaríamos a cabo. Al detectar un animal peligroso, entonces, el miedo incrementará nuestra disposición a evaluar a animales similares como 
amenazas. La detección de una situación peligrosa modula el modo en que caracterizamos las cosas como peligrosas o no peligrosas. Al menos en esta dimensión de la respuesta emocional encontramos, dentro del enfoque evolutivo, una forma de auto-regulación.

El enfoque evolutivo da lugar a diferentes formas de regulación emocional que no requieren la presencia de programas de orden superior. Por supuesto, esto no quiere decir que el enfoque sea incompatible con este tipo de programas ni que no pueda haber una motivación legítima para introducirlos. El punto es que dicha motivación no se encuentra en el interior del enfoque evolutivo. El tipo de interferencia que motiva la introducción de los programas de orden superior que son las emociones no se reproduce en este segundo nivel. El conflicto se resuelve de manera lateral.

\section{Conclusión}

En este trabajo discutí la presunta independencia de la noción de regulación emocional respecto de la teoría de emociones subyacente. En particular, sostuve que adoptar un enfoque evolutivo conlleva una serie de consecuencias para la caracterización de la relación entre emoción y regulación emocional. En primer lugar, sostuve que el enfoque implica que los tipos de procesos que se consideran y estudian comúnmente como instancias de regulación emocional son en realidad componentes de las emociones mismas. Es decir, la propuesta implica que la línea entre emoción y regulación emocional está mal trazada.

En segundo lugar, sostuve que el enfoque evolutivo implica que al menos una parte importante de la regulación emocional no se lleva a cabo verticalmente, por medio de procesos de segundo orden, sino lateral $\mathrm{u}$ horizontalmente, entre diferentes emociones que se encuentran en el mismo nivel. En particular, sostuve que el argumento de la interferencia entre procesos incompatibles no puede usarse para postular procesos reflexivos que regulen a las emociones ya que estas mismas incluyen comandos para desactivar programas emocionales alternativos.

Mi intención en este trabajo no fue defender el enfoque evolutivo sobre 
las emociones, sino más bien tomarlo como caso particular para cuestionar la presunta neutralidad de la noción de regulación emocional de Gross. Las decisiones teóricas que tomemos para entender a las emociones pueden tener impacto sobre nuestra teoría de la regulación emocional. De esta manera, no podemos defender un enfoque particular sobre la última sin discutir previamente la caracterización de la primera.

\section{Referencias bibliográficas}

- Arnold, M. B. (1960), Emotion and personality. Volume I: Psychological aspects, New York, NY, Columbia University Press.

- Berridge, K. C. (2009) Wanting and liking: Observations from the neuroscience and psychology laboratory. Inquiry 52, pp. 378-398.

- Cosmides, L. \& Tooby, J. (2000), "Evolutionary psychology and the emotions", en Lewis, M \& Haviland-Jones, J. M. (Eds.), Handbook of emotions (2nd ed.), New York, Guilford Press, pp. 91-115.

- Cosmides, L. \& Tooby, J. (2013), "Evolutionary psychology: New perspectives on cognition and motivation", Annual Review of Psychology, 64, pp. 201-229.

- Georgiadis, J. R., \& Kortekaas, R. (2010), “The sweetest taboo: Functional neurobiology of human sexuality in relation to pleasure", en M. L. Kringelbach \& K. C. Berridge (Eds.), Pleasures of the brain. New York, NY, Oxford University Press, pp. 178-201.

- Gross, J. J. (2015), "Emotion regulation: Current status and future prospects", Psychological Inquiry 26, pp. 1-26.

- Gross, J. J. \& Thompson, R. (2007), "Emotion Regulation: Conceptual Foundations", en Gross, J. J. (Ed.) Handbook of emotion regulation, New York, Guilford Press, pp. 3-24.

- Kappas, A. (2011), Emotion and Regulation are One!, Emotion Review, 3 (1), pp. 17-25.

- Keltner, D., Haidt, J., \& Shiota, M. N. (2006), "Social functionalism and the evolution of emotions”, en Schaller, M., Simpson, J. A., Kenrick, D. T. (Eds.), Evolution and social psychology, New York, Psychology Press, pp. 115-142.

- Nesse, R. (1990), "Evolutionary explanations of emotions", Human Nature, 1, pp. 261-289. 
- Richardson, R. C. (1996), "The prospects for an evolutionary psychology: Human language and human reasoning", Minds and Machines, 6, pp. 541-557.

- Rothbart, M. K., \& Sheese, B. E. (2007), "Temperament and emotion regulation", en J. J. Gross (Ed.), Handbook of emotion regulation, New York, Guilford Press, pp. 331-350.

- Stifter, C. A., \& Moyer, D. (1991), "The regulation of positive affect: Gaze aversion activity during mother-infant interaction", Infant Behaviors and Development, 14, pp. 111-123.

- Sznycer, D, Cosmides, L \& Tooby, J. (2017), "Adaptationism Carves Emotions at Their Functional Joints”, Psychological Inquiry, 28, pp. 56-62.

- Tooby, J., \& Cosmides, L. (1990), "The past explains the present: Emotional adaptations and the structure of ancestral environments", Ethology and Sociobiology, 11, 375-424.

- Tooby, J. \& Cosmides, L. (2008), “The evolutionary psychology of the emotions and their relationship to internal regulatory variables", en Lewis, M., Haviland-Jones, J. M. \& Barrett, L. F. (Eds.), Handbook of Emotions, 3era Ed., NY, Guilford, pp. 114-137.

- Watts, F. (2007), "Emotion regulation and religion", en J. J. Gross (Ed.), Handbook of emotion regulation. New York: Guilford Press, pp. 504-520.

Recibido: 05/2018; aceptado: 08/2108. 